\title{
Development of a Socially Believable Multi-Robot Solution from Town to Home
}

\author{
Filippo Cavallo - Raffaele Limosani - Alessandro Manzi - Manuele Bonaccorsi • \\ Raffaele Esposito • Maurizio Di Rocco - Federico Pecora - Giancarlo Teti • \\ Alessandro Saffiotti • Paolo Dario
}

Received: 20 December 2013/ Accepted: 20 June 2014/Published online: 3 July 2014

(C) Springer Science+Business Media New York 2014

\begin{abstract}
Technological advances in the robotic and ICT fields represent an effective solution to address specific societal problems to support ageing and independent life. One of the key factors for these technologies is that they have to be socially acceptable and believable to the endusers. This paper aimed to present some technological aspects that have been faced to develop the Robot-Era system, a multi-robotic system that is able to act in a socially believable way in the environments daily inhabited by humans, such as urban areas, buildings and homes. In particular, this paper focuses on two services-shopping delivery and garbage collection-showing preliminary results on experiments conducted with 35 elderly people. The analysis adopts an end-user-oriented perspective, considering some of the main attributes of acceptability: usability, attitude, anxiety, trust and quality of life.
\end{abstract}

Keywords Service robotics - Social robotics - Multirobot cooperation - Smart environments - Ambient-assisted living

F. Cavallo $(\bowtie) \cdot$ R. Limosani · A. Manzi · M. Bonaccorsi ·

R. Esposito · P. Dario

BioRobotics Institute, Scuola Superiore Sant'Anna, Pisa, Italy

e-mail: f.cavallo@sssup.it

M. Di Rocco · F. Pecora · A. Saffiotti

Center for Applied Autonomous Sensor Systems (AASS),

Orebro University, Örebro, Sweden

e-mail: asaffio@aass.oru.se

G. Teti

Robotech Srl, Peccioli, Italy

e-mail: g.teti@ robotechsrl.com

\section{Introduction}

Due to recent advances in the field of service robotics, the range of potential applications for robots has greatly expanded [1], becoming one of the most emerging technologies devoted to helping and assisting citizens in daily activities at home, in their workspaces and in other environments [2]. Among several applications in the fields of defence, rescue, security, healthcare and agriculture [3], service robotics have been conceived to address specific societal problems and market opportunities as advanced service and social robotics in support of ageing, independent life, work, social innovation and inclusion. Indeed, recent demographic changes, due to the increase in life expectancy and the reduction of births, are leading to an increase in the old-age dependency ratio [4]. Considering this social concern, technological advances in the robotic and ICT fields represent an effective solution to address societal trends and opportunities related to the ageing population. As a matter of fact, different approaches have been proposed to develop solutions. With these technologies, it is possible to provide smart services, improving citizens' daily life and reducing health and social costs.

Assisting elderly people at home is one of the principal needs that must be addressed. For this reason, previous studies in this field have focused on robots that primarily help and assist people in daily activities at home. Such robots are usually named "Robot Companion" [2]. Beyond the assistive aspect, social acceptability also plays an important role. Examples of robots used to enhance social inclusion can be found in iCat [5], Paro [6] and Huggable [7].

Robotic service solutions range from the simplest telepresence functionalities to support caregivers, such as the Giraff [8] developed in the ExCITE project [9], AVA [10] and Luna [11], to the most complex, such as assistance for 
daily living activities [12], self-management of chronic diseases [13], well-being and safety as in the cases of Florence [14] and Robo M.D. [15], and integration in a smart environment $[16,17]$.

Most of these projects concern robotic solutions for home applications. Very few robotic applications deal with social services in other environments, such as the garbage collection performed by DustCart [18], assistance in shopping centre [19] and smart office building [20, 21].

In this paper, we describe the progress achieved in the Robot-Era project [22], which aims to develop, implement and demonstrate the general feasibility, scientific/technical effectiveness and social/legal plausibility and acceptability by end-users of a plurality of complete advanced services by means of a multitude of cooperating robots integrated with smart environments and acting in heterogeneous environments, such as the home, a condominium and outdoors.

It is clear that one of the most important aspects for a complex robotic system that aims to reach such ambitious objectives is the need to be socially acceptable and believable by the end-users. The current design and development of the system are based on a considerable study carried out with elderly people and caregivers using a user-centred design approach that allowed us to identify needs, services and scientific and technological requirements in order to develop adequate solutions [23]. Detected services include transporting/manipulating objects at home, cleaning, garbage collection, surveillance, outdoor walking support, indoor escorting at night and reminding events, laundry support, communicating with people, food delivery and shopping/drug delivery. Some of the above services have already been tested with 35 elderly people. In particular, this paper focuses on the shopping delivery and the garbage collection scenarios and presents preliminary results in terms of attitude towards and usability and generally acceptability of both the robots and the services. The implementation and experimentation of these two services required solutions to different technological issues, including task planning, human-robot interaction, user localisation and the use of three different robots able to cooperate for exchanging objects and to navigate through different floors interfacing with an elevator.

This paper aimed to present some technological aspects that have been encountered in developing a robotic system that is able to act in a socially believable way and is suitable for use in the environments daily inhabited by humans, such as urban areas, buildings and homes.

The key topics treated in this work concern the efficiency of the robotic system in physically approaching the user, so that the user perceives that the service is provided:

1. The physical interaction of the robot with humans is improved if the robot is able to approach the human

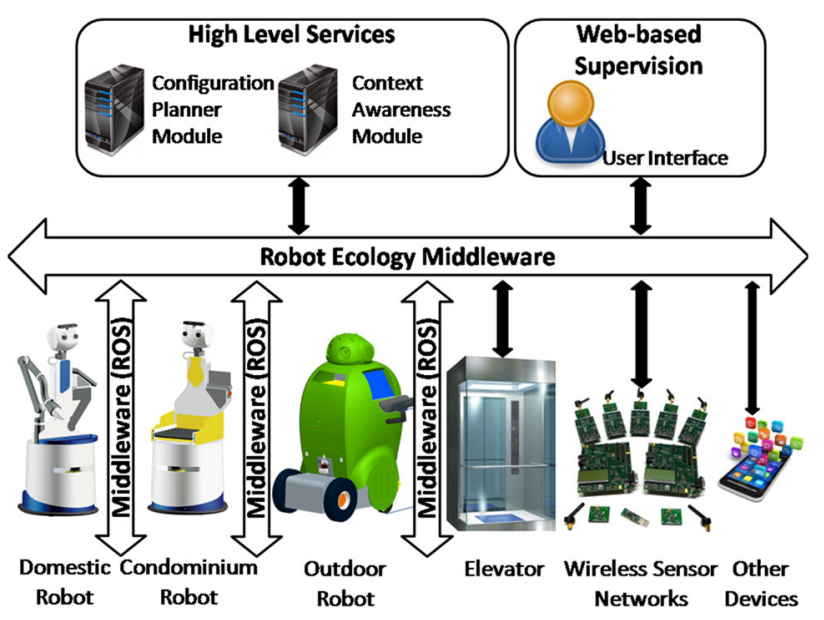

Fig. 1 Architecture of the Robot-Era system. At the bottom, there are all the agents, such as robots, elevator and sensors. At the top of the system, there are the Context Awareness Module, the Configuration Planner Module and the user interfaces

target efficiently by knowing in advance his/her location. This is particularly important to avoid the need for the robot to discover the human target in the environment by using sophisticated sensors and a high computational load.

2. The integration of the robotic systems with the capability to interact and cooperate with common agents (elevator, different floors, etc.) is necessary. Thus, a planner is of great importance.

3. The capability of the system to cover the entire service chain, from town to home and vice versa is essential. Thus, robot cooperation is important.

The paper is organised as follows. In the next section, the overall architecture of the system is shown. In Section three, the involved agents are described. Then, in Section four, the Context Awareness Module (CAM) and the user localisation procedure are detailed. In Section five, the Configuration Planner Module (CPM) is explained and an example of the generated plan is shown. Section six details the shopping delivery and garbage collection scenarios, while Section seven and Section eight deepen the adopted strategy for the multi-map navigation and the docking procedure used for the "goods exchange" task. Finally, experiments with elderly users are described (Section nine) and some results detailed (Section ten). In Section eleven, conclusions of the work are presented.

\section{Overall Architecture}

The Robot-Era architecture integrates a multi-robot system able to work in different environments such as outdoors, condominium and homes. It also includes a domestic 
wireless sensor network (WSN), constituting an ambient intelligence (AmI) infrastructure that supervises the home and localises the user. Other agents of the system include the elevator and the user interface sub-system (i.e. tablet and microphone).

The architecture is structured into four abstract layers (see Fig. 1):

\section{Hardware and Low-Level Control}

The system is composed of heterogeneous devices, and in the current configuration, it includes three different robots acting in three different environments: domestic, condominium and outdoors. It also includes several sensors, such as ZigBee boards and pressure and presence sensors, constituting the WSN.

\section{Local Control}

Every agent has its specific control software for local control integrated in the system. The robotic platforms, for example, are based on the robot operating system (ROS) [24], while specific agents, such as the elevator or the WSN, use ad hoc solutions. The agents communicate with each other using a common middleware (see below).

\section{PEIS Middleware}

The communication among all the agents relies on the PEIS middleware [25], a distributed blackboard-based infrastructure in which different devices communicate in a uniform way. The fully decentralised nature of this middleware allows the system to be easily extended and does not have a central point of failure. The whole system constitutes the so-called PEIS Ecology.

\section{Global Control}

At the top of the system, there are three main components: the CAM, which maintains knowledge about the status of all the system modules, the CPM, which manages and coordinates the system modules in order to achieve services, and the web-based user interface used to request services. Services can also be generated autonomously by the system if the CAM detects a particular or critical situation such as a gas leak.

\section{Agents of the Ecology}

In this section, some details about the agents of the ecology will be provided. In order to be able to operate in different

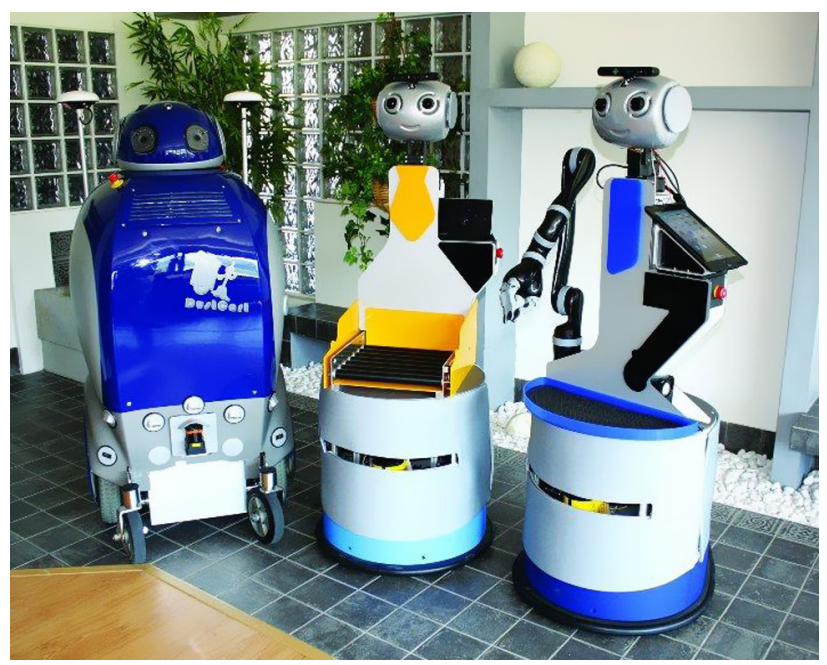

Fig. 2 Robot-Era robots, from left to right Oro, Coro and Doro

environments, three mobile robots (see Fig. 2), each with its own characteristics, are used. The Domestic (Doro) and Condominium (Coro) robots share most of their hardware components and they were designed upon the SCITOS G5 mobile platform (developed by Metralabs [26]). The outdoor robot (Oro) is the DustCart platform [27], whose first version was produced within the DustBot project (Networked and Cooperating Robots for Urban Hygiene, FP6-045299, 2006-2009).

The local control software of the robots is implemented as ROS nodes, though the navigation stack of the SCITOS platforms (Doro and Coro) relies on CogniDrive, a proprietary software developed by Metralabs that is encapsulated in a ROS node.

\section{Doro}

The domestic robot has to navigate safely in a domestic environment. Mounted on the robot are a front laser (SICK S300) and a rear laser (Hokuyo URG-04LX) in order to give Doro a $360^{\circ}$ field of view for avoiding obstacles and for self-localisation. The robot dimensions are $160 \mathrm{~cm} \times 60 \mathrm{~cm} \times 60 \mathrm{~cm}(\mathrm{H} \mathrm{x} \mathrm{L} \mathrm{x} \mathrm{W})$ with a weight of about $50 \mathrm{~kg}$. A Kinova Jaco arm is used for manipulation tasks. The head of the robot has a pan-tilt unit, an Asus XtionPro and high-resolution cameras used for object detection. Multicolour LEDs, mounted on the eyes, and speakers provide feedback to the user. The robot brings a removable tablet that the user can use for service requests. Motion planning is based on the dynamic window approach [28], while localisation relies on the adaptive Monte Carlo localisation technique [29]. The indoor localisation system has an accuracy of around 3-5 cm. 


\section{Coro}

The condominium robot has to navigate between floors via the elevator. Most of the hardware and software is shared with Doro. It does not have an arm, but it mounts a roller mechanism in order to be able to exchange goods with Oro.

Oro

The outdoor robot is an autonomous mobile robot designed to transport objects in an urban environment. The robot consists of a mobile base, a container for the objects, a robotic head, a touch screen used primarily for human-robot interaction and sensors for obstacle detection and localisation.

The mobile base consists of a mechanical chassis with two central actuated wheels (Swiss drive $400 \mathrm{~T}$ hub motor with electromagnetic brake and encoder by Micro-Motor AG) and four passive rear wheels with shock absorbers. The robot dimensions are $150 \mathrm{~cm} \times 100 \mathrm{~cm} \times 80 \mathrm{~cm}(\mathrm{H} \times \mathrm{L} \times \mathrm{W})$ with a weight of $150 \mathrm{~kg}$. The container is actuated by a motor and can be opened/closed on the rear part of the robot. The touch screen, mounted on the left side of the robot, multicolour LEDs in the eyes and speakers reproducing acoustic signals and recorded vocal messages provide information to the user about the state of the robot. The sensors for obstacle detection consist of a laser scanner (Hokuyo UTM-30LX) positioned on the front of the robot and of infrared and ultrasound sensors used to detect steps, sidewalks, road gaps and any other common obstacle in the urban environment. The localisation system consists of two GPSs and two antennas (provided by NOVATEL) mounted on the back of the robot providing robot position and orientation with an accuracy of $5 \mathrm{~cm}$ and $1^{\circ}$ with differential correction applied. The local control system of the robot is composed of many software modules running on the main PC and integrated into the ROS.

\section{User Interface}

The user can interact with the system using a web-based interface, runnable from the Doro tablet or any smartphone, or with a wearable microphone connected to a speech recognition software module.

\section{Domestic Sensors}

The sensors placed in the home environments are based on a ZigBee-Pro low-cost, small-form-factor, general-purpose radio module. The same boards are used to compose the sensor network for both environmental monitoring and the user localisation network, depending on the firmware uploaded (see Sec. IV). In particular, the Ember ZNet ZigBee-Pro stack is used as a communication stack for these two networks. The board, its sensors and its functional parts are shown in Fig. 3.

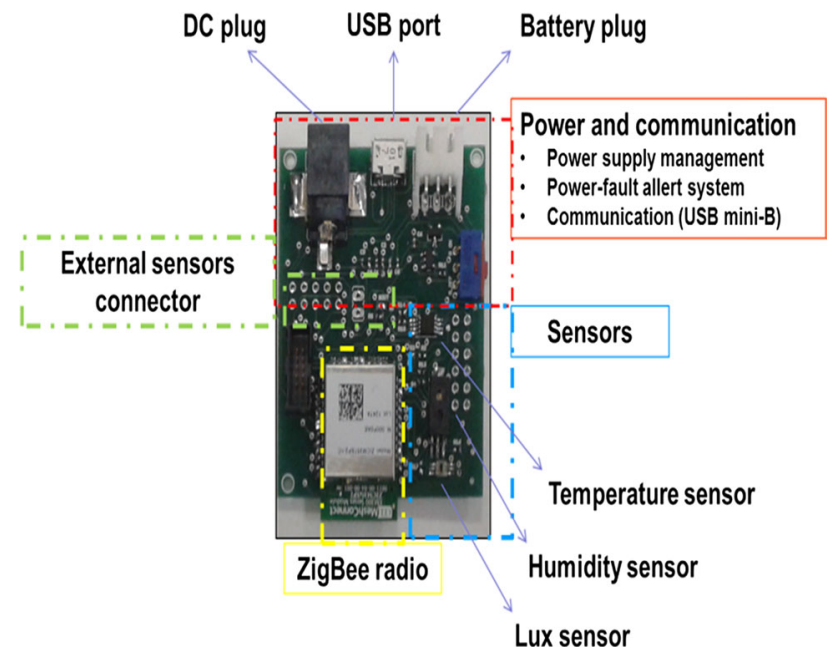

Fig. 3 Robot-Era ZigBee board used to set the Localisation Network and the Service Network

Each board is equipped with a CEL's MeshConnect ${ }^{\mathrm{TM}}$ EM357 Mini Module (CELL, USA) that is composed of a ZigBee radio module and a power amplifier to extend the radio range. The power management system allows the board to be powered from a $5 \mathrm{~V}$ DC plug or a USB mini-B port, while a lithium-ion battery is used for power backup. A dedicated microchip is embedded to recharge the battery and act as an emergency power system. Three sensors are embedded for environmental monitoring: a digital temperature sensor, an analogical humidity sensor and a digital light sensor. An external antenna connector allows sectorial antennas to be plugged into spot-specific workspace areas, improving localisation accuracy. A pin header provides connection to six GPIO ports and a power supply and ground to plug in external sensors such as PIRs (Passive InfraRed) and switches.

\section{Elevator Control Unit}

A Koala Mini PC equipped with an Atom $1.8 \mathrm{GHz} \mathrm{WiFi}$ connection and a Phidget Input/Output digital board are used to control the elevator remotely. The local control software that runs on the elevator is composed of:

- a TCP server that enables control of the elevator functionalities, such as going to a specific floor, opening/closing the door and keeping the door open;

- a TCP client connected with the ecology, allowing integration with the PEIS middleware.

\section{Context Awareness Module and User Localisation}

Context awareness is an important asset of the Robot-Era system in order to provide localisation-based services to 
users. The system continuously monitors several parameters related to the state of the user and of the environment. The CAM was developed to estimate the home status and the user position, making them available to the whole PEIS Ecology. It fuses heterogeneous information from a set of WSN nodes installed in the home, and worn by the user. This set-up provides an unobtrusive localisation and monitoring service that complies with privacy issues [30, 31]. The WSNs are also designed to cover the whole condominium environment thanks to the multi-hop communication provided by the ZigBee-Pro stack.

\section{WSN Design}

The main source of context information is the WSN, composed of two parts: the Localisation Network (LNet) and the Service Network (SNet). The LNet is designed for multiple user localisation using a Received Signal Strength Indicator (RSSI). It is composed of a ZigBee Coordinator (ZC), a Data Logger (DL), a wearable Mobile Node (MN) and a set of ZigBee Anchors (ZAs). The MN periodically sends messages to all the ZAs within one communication hop. Each ZA computes the RSSI of the received messages and sends this value to the DL. The SNet is developed for home monitoring and passive localisation of people. It is composed of a ZC, a DL and a set of Sensor Nodes (SNs). Each SN contains a selection of sensors, such as PIR, proximity sensors, a pressure sensor (Press) placed under a chair or a bed, switches on doors or drawers, and temperature, humidity and light sensors. The LNet and SNet are set on different channels to avoid interferences. Each DL node is connected to a PC via a USB. The LNet and SNet design in the DomoCasa Lab (Peccioli, IT) are shown in Fig. 4. In order to improve acceptability of the localisation system, a necklace integrates the MN. The MN automatically configures itself, connecting to the LNet and sending messages to the neighbouring ZAs without the need for configuration. A blinking blue LED reports the running state of the MN and whenever the user demands privacy, a switch on the node can be used to stop the localisation service.

\section{Localisation Algorithm}

One of the most important parameters for the Robot-Era system is the location of people. This parameter is estimated by the CAM, which performs sensor fusion on the data from the LNet and SNet. The localisation system is designed taking into account the state of the art in indoor localisation systems for ambient-assisted living [30]. Area-based localisation methods [32], trilateration [33] and presence detection are combined to provide a room-level user position estimation. The entire

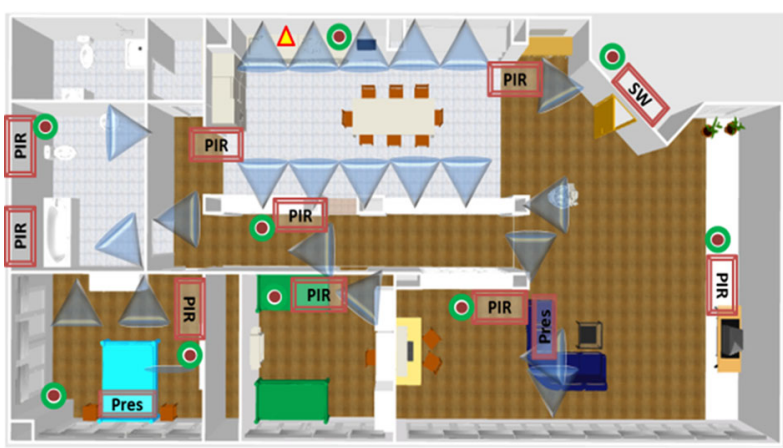

ZigBee anchors for user localization and antenna workspace

Proximity Infrared Sensors (PIR), Switches (SW) and Presence sensors (Press)

(-) Temperature, Humidity and light sensors

$\triangle \quad$ GAS sensor

Fig. 4 Service Network and Localisation Network design in the DomoCasa Lab (Peccioli, IT)

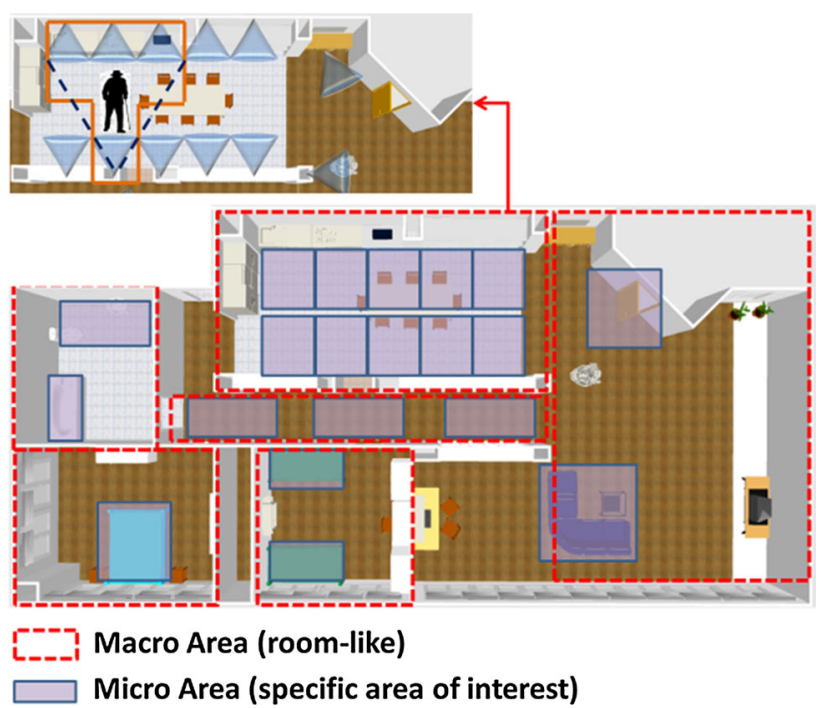

Fig. 5 An example of micro- and macro-localisation areas in the DomoCasa Lab (Peccioli, IT)

workspace is divided into room-like macro-areas, each one including at least one SNet sensor and one LNet anchor. Each macro-area comprises one or more microareas representing specific areas of interest for user localisation and corresponding to the sensing range of a $\mathrm{ZA}$ or a SN. For a ZA, the range is given by the projection of the antenna radiation lobe in the workspace (Fig. 5). For each ZA, a Path Loss model and a RSSI threshold are computed in the calibration phase. For simplicity, a linear model is used, as suggested in Whitehouse et al. [34]. The RSSI threshold is set to the value measured two metres away from the point of 


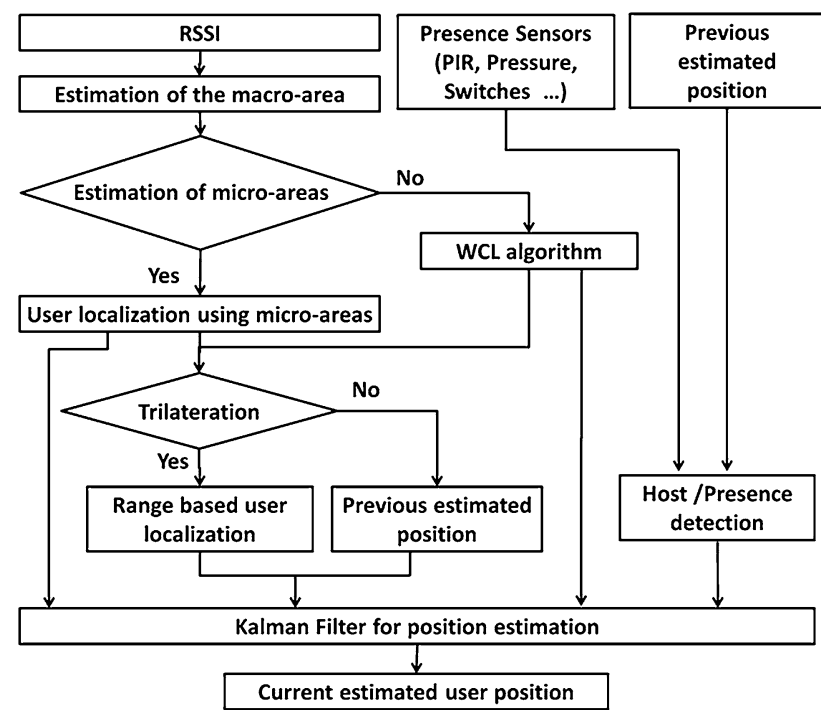

Fig. 6 Localisation algorithm using a top-down approach and sensor

highest RSSI. Localisation is done using a top-down approach, from home to room level, as shown in Fig. 6.

Initially, the location is set to the macro-area where at least one ZA observes an RSSI value over the threshold. If more than one such macro-area exists, the one from the previous time point is selected. For each ZA of this kind, the centre of mass of the corresponding micro-area is considered: the location of the $\mathrm{MN}$ is estimated to be on the median of those. If no ZA has a RSSI value over the threshold, a Weighted Centroid Localisation algorithm is used [32], in which the weight of each ZA is computed directly from the observed RSSI without the need for distance estimation to reduce the computational complexity.

Then, a trilateration technique is used to estimate the MN position using the distances calculated from the four neighbouring ZAs, which is better able to spot with the antenna the micro-area where the user is located.

Finally, a Kalman filter is used to fuse the RSSI-based estimate provided by the above algorithm with the presence information provided from the SNet through the PIR and pressure sensors.

\section{Configuration Planner Module}

The heterogeneity of the components involved in the system requires a form of sophisticated reasoning: the tasks typically required can be accomplished in different ways depending on the specific state of the environment; they are in general dynamic, which is to say that the human user can post them anytime, also implying concurrency between multiple goals; and other requirements can even be generated by the system itself monitoring the state of the system (e.g. a gas sensor could trigger the intervention of a robot to notify the user). Furthermore, a task execution often requires a set of interconnected (and heterogeneous) actions carried out by a multi-robot system in which the access to shared resources (e.g. a condominium robot supporting the activities related to multiple apartments) must be carefully managed.

This interconnection introduces several points of failure, and therefore, the system must also be able to react (i.e. change over time) to unexpected contingencies. The CPM copes with these needs: it produces configuration plansthat is, fine-grained action plans that specify the actions of all the components in the Robot-Era system, as well as the information flow among them [35]. The CPM takes into account information, resource and temporal constraints. Each of these aspects is managed by a specialised solver that acts on a common temporal network [36] in order to solve possible conflicts. Although the temporal aspect is just one of the facets addressed in the reasoning process, it is of particular interest in our application since many userrelated tasks may involve temporal bounds (e.g. reminders), and since temporal prediction helps properly manage the available resources.

A distinctive feature of our planner is its closed-loop performance: new observations coming from the robots and from the CAM are continuously incorporated into the plan, which is therefore modified if needed. Modifications are kept to a minimum, avoiding replanning from scratch when possible [37]. This feature highly enhances the robustness of the plan in face of unexpected contingencies. Such a structure can be easily associated with a closed-loop system in which goals play the role of desired states, in our case a particular configuration of the world that must be reached. To do that our planner acts like a controller injecting control signals (i.e. deploying activities) that over time are modified, taking into account the feedback gathered by sensor readings. Such a structure is depicted in Fig. 7.

In particular, the generation of the plan relies on the following solvers:

\section{Temporal Solver}

The temporal consistency of the constraint network is checked through temporal constraint propagation by means of a simple temporal problem (STP) solver [36]. The solver propagates temporal constraints to refine the bounds of the activities in the network and returns failure if and only if temporally consistent bounds cannot be found.

\section{Resource Scheduler}

This solver ensures that resources are never over consumed. The maximum capacities of resources restrict 




Fig. 7 High-level reasoners (causal reasoner, information dependency reasoner and schedulers) modify the constraint network so as to achieve the dynamically changing desired state (dynamic goal posting). Their decisions are temporally validated (temporal reasoning) and sent to the system as control signals. Reasoning accounts for the current state of the system, which is continuously maintained in the constraint network (observer)

which activities can occur concurrently, and this solver posts temporal constraints to the constraint network, ensuring that over-consuming peaks of activities are avoided [38].

\section{State Variable Scheduler}

State variable scheduling ensures that activities do not prescribe conflicting states in overlapping intervals. Similar to the resource scheduler, this solver posts temporal constraints, which impose a temporal separation between conflicting activities.

\section{Information Dependency Reasoner}

Operators model the information dependencies between functionalities. This solver instantiates into the constraint network relevant operators (in the form of activities and temporal constraints) so as to enforce the information dependencies.

\section{Causal Reasoner}

Operators in the domain also model causal dependencies between states. This solver instantiates into the constraint network relevant operators (in the form of activities and temporal constraints) so as to enforce the causal dependencies of the configuration plan.

Each of solvers is able to intervene in posting new activities or constraints in order to solve all the dependencies and conflicts that may appear at planning or execution time. In the latter case, inconsistencies may arise from sensor readings (e.g. a planned activity has to be postponed given that the current state of the environment does not allow execution) or from new goals, which require the fulfilment of new dependencies. The interleaving and ordering mechanisms by which these solvers act on the temporal network are grounded in heuristic methods whose details are discussed by Di Rocco et al. [37]. Figure 8 shows an example of the plan generated by the CPM-namely, the one for the shopping delivery scenario. The plan prescribes the activities over time of the three robots and of the elevator. Initially, Oro is commanded to reach the shop, take the goods from the shopper and come back to the entrance; meanwhile, Coro is commanded to reach the entrance of the building (labelled as ENT in Fig. 8). Since Coro started from the first floor, the CPM manages the position of the elevator and guarantees that the elevator's door remains open while Coro is entering or exiting the elevator.

When both Coro and Oro are at the entrance of the building, the planner starts a coordinated "exchange" action. When the exchange is completed, Coro is moved back to the elevator. The elevator is sent from floor F0 to floor F1, and then, Coro is sent to the user's apartment. When it arrives, the domestic robot Doro is moved to the user's location, which is computed by the CAM, and notifies him/her that Coro is waiting at the door with the groceries. The task is completed after the user has interacted with Coro to confirm that he/she has taken the groceries. This plan exposes a couple of interesting points. First, the planner can generate both parallel activities which are loosely coupled, such as the initial MoveTo activities of Oro and Coro, and activities which are tightly coupled, such as the two transfer activities. In the latter case, the planner posts constraints to ensure that the two activities overlap in space and time. The coordinated execution of these activities is implemented through a local synchronisation mechanism. Second, the planner plans movements using not only topological information, but also causal and temporal information. For instance, the plan to move Coro from the entrance (ENT) to the apartment (HOME) incorporates the coordinated use of the elevator and the operation of the doors at the right moments. As for the transfer activities, the fine-grained synchronisation of these activities is delegated to the execution mechanism. Finally, each interaction with the user is treated by the planner as just another activity, such as the notification or confirmation activities. These are performed in tight coupling between the robot and a human with interaction-specific modalities.

\section{Shopping Delivery and Garbage Collection Services}

Within the Robot-Era project, some services were identified starting from the outcome of the study performed in Italy, Germany and Sweden on users' needs [23]. This study is based on the User Design Approach, in which the end-users were involved not only during the evaluation 




Fig. 8 Example of the plan generated for the shopping delivery service

phase, but also during the designing stage and through all the development phases of the services. In this section, we focus on two of the most complex services developed and tested in the current configuration of the Robot-Era project: shopping delivery and garbage collection. These services are considered useful by 75 and $62 \%$, respectively, of the people involved during the end-users' needs analysis.

The complexity of the implemented services is distributed among different agents of the system working in different areas of the environments involved (home, condominium and outdoors).

\section{Drug and Shopping Delivery}

The aim of this service is to provide a complete means of delivering groceries from the shop to the user's apartment. The service starts with a user's request through a voice command using the wearable wireless microphone. Doro autonomously navigates towards the current location of the user, exploiting the information generated by the CAM. Using the web-based interface running on the robot tablet, the user selects the desired service and makes a shopping list starting from a list of everyday goods. This list is sent to the proper shop and the CPM generates a plan for the service. After the dispatch of the list, Oro autonomously navigates to the grocery shop. Using Oro's touch screen, the marketer loads the goods inside the robot. Then, Oro moves to the condominium and, more precisely, to the "goods exchange location". Coro waits until Oro indicates that it is ready for the exchange procedure. This task is detailed in Robot Co-operation" section. When the condominium robot has the pack, it navigates through the building using the elevator (see "Multi-Map Navigation" section). When it arrives at the user's apartment, Doro notifies him/her that Coro is waiting at the door. The user takes the groceries from Coro and says "thank you", at which point the service ends and Coro returns to its default position.

\section{Garbage Collection}

The aim of this service is to provide a complete way to collect garbage from the home of the user. The user requests the "garbage collection" service by means of the tablet or the microphone. Choosing the service, the CPM plans the proper activities. Coro moves to the user's apartment; when the robot arrives at the door, Doro tells the user that Coro has arrived. Then, the user loads the garbage onto the robot and confirms the action using a vocal interaction (saying "thank you"); from the point of view of the user, the service is concluded at this point. After the loading of the garbage, Coro autonomously moves to the entrance of the building; meanwhile, the Oro has been moving towards the entrance as well. As described in the shopping delivery service and technically in Robot Co-operation" section, the two platforms exchange the item by a system with an actuated roller. Finally, Oro autonomously navigates to a predefined discharge point and unloads the garbage.

The main objective of the implemented scenarios is to provide a complete service running in real everyday environments. The overall implementation also aims to simplify, where possible, the use of the service by non-expert users.

\section{Multi-Map Navigation}

The Oro and Coro robots are designed to work outdoors and in a condominium, respectively; hence, the robots need multiple maps. While the first one uses multiple outdoor maps to be able to navigate in broad areas, the second one 
has to move on and between different floors via the elevator. The global control system creates topological paths through the different areas and, as explained in "Configuration Planner Module", manages the interactions with the smart environments (e.g. elevator and actuated doors). The local control system of the robot translates the topological primitives into suitable commands such as map switching if needed. Since the elevator door and its inner space are very narrow ( $80 \mathrm{~cm}$ wide and less than $1 \mathrm{~m}^{2}$, respectively), an ad hoc strategy for entering/exiting the elevator was adopted. The local control system is able to adjust dynamically specific navigation parameters, such as the maximum navigation speed, the yaw goal tolerance and the preferred direction of navigation. To ease the entering/ exiting elevator task, the strategy generally adopted is to avoid rotation when the robot is close to the elevator.

\section{Robot Co-operation}

Implementing a service which operates in different areas implies problems within the "link" between heterogeneous environments. The solution used in the Robot-Era system is based on the division of workspaces among the different agents involved in the system. Specifically, during the implementation of the shopping delivery and garbage collection services, the main problem occurred while carrying items from an outdoor environment to an indoor one and vice versa. In more detail, the haulage of goods is managed through an exchange phase between Coro and Oro. From a hardware point of view, the exchange is performed through actuated rollers on mobile platforms-the SCITOS G5 platform and the Oro robot (Fig. 9) - and the mechanism of the mobile cart in Oro is also used to open and close the basket.

The dimensions of the roller conveyer platforms are approximately $320 \mathrm{~mm} \times 320 \mathrm{~mm}$, and the weight is around $5 \mathrm{~kg}$ each. The basket dimension is $25 \mathrm{~cm} \times$ $30 \mathrm{~cm} \times 50 \mathrm{~cm}$. The actuation and the status of the roller are performed by dedicated ROS nodes acquiring data via the CAN bus already present on the two platforms. From an algorithmic point of view, the task is managed using a finite state machine implemented as an ROS node on the two platforms; the information between robots is exchanged using the PEIS middleware but, unlike the other tasks, the flow of operations is controlled directly by the platforms without the supervision of the CPM: at the planner level, the action is represented as a unique "goods exchange_task". With regard to the shopping service, both Coro and Oro navigate to the exchange point and start the exchange goods task triggered by the CPM. Next, Oro opens its basket and orders Coro to start the docking phase.


Fig. 9 Roller on Oro and the SCITOS G5 platform

The docking represents the way used by the Coro platform to approach the basket of Oro closely; it is based on the Cognidrive's docking function formerly implemented to move the SCITOS G5 close to the recharging station easily identified through a characteristic shape. In the case of docking between two mobile platforms, two main drawbacks occurred:

- in the Cognidrive's docking function, the docking station is in a fixed pose rather than on a mobile platform;

- the shape of Oro has less identifying characteristics than the docking station.

To overcome these difficulties, different strategies were tried; the actual solution is depicted in Fig. 10. The Oro moves to a preset docking position, but due to navigation tolerance and localisation error, the actual docking position is different from the preset one; to facilitate the docking procedure, Coro is sent in front of the actual position of Oro in advance. The goal of navigation is based on the offset sent through the PEIS communication middleware and is managed in the condominium map frame using the ROS Transformation Frame tool. After the docking phase, the rollers are turned on, starting with the platform that is receiving the package; the end of the transfer is managed without communication between platforms due to the integrated infrared sensors, which recognise the transition of the goods. At the end of the task, Coro docks off and only after this event the basket is closed: in this way, contact between the closing cover of the basket and Coro is avoided.

\section{Experimentation}

The experimentation was conducted in Peccioli (Italy), where elderly participants were invited to interact with the robots in order to evaluate the technical effectiveness and acceptability of the Robot-Era robotic services. 


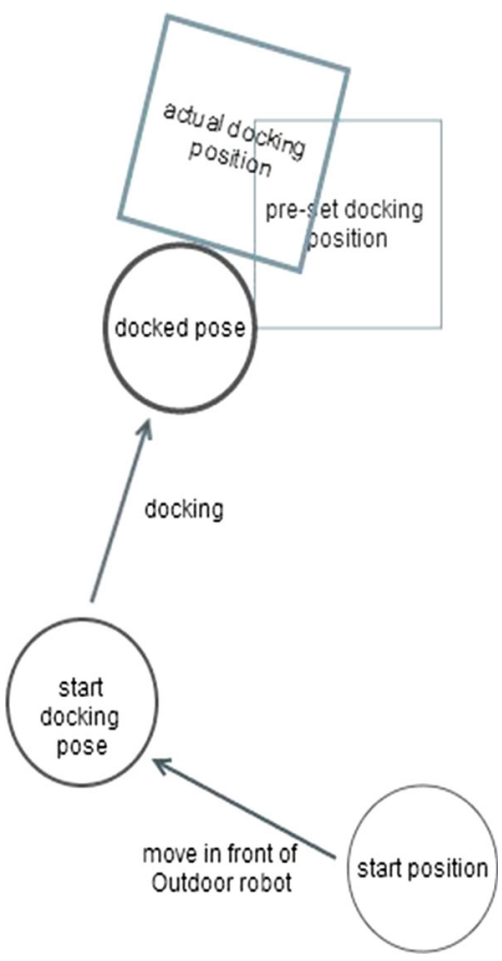

Fig. 10 Docking strategy used

\section{Experimental Set-up}

As described in "Shopping Delivery and Garbage Collection Services" section, the overall system works in three different environments:

- domestic: the DomoCasa Lab, a domotic house developed and managed by the BioRobotics Institute of Scuola Superiore Sant'Anna in Peccioli (Italy) (Fig. 11);

- condominium: common areas, such as the entrance hall, corridors and elevator, of the building where the DomoCasa Lab is located;

- urban: the surrounding outdoor pedestrian area; also addressed were the legal and bureaucratic issues associated with obtaining an Agreement with the Municipality of Peccioli for experimentation in the devoted urban area.

\section{Participants}

The inclusion criteria to participate in the study were as follows:

- age $\geq 65$ years;

- positive evaluation of mental status with the SPSMQ [39];

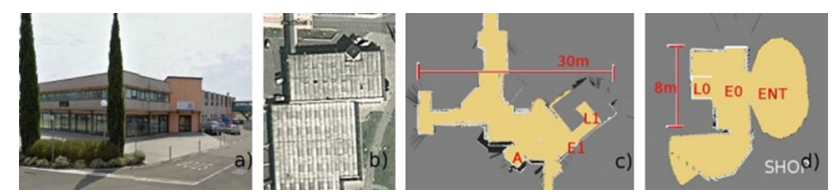

Fig. 11 Pilot site: a DomoCasa Lab, b Satellite image of the DomoCasa Lab, and Cognidrive's map of the first floor (c) and the ground floor $(\mathbf{d})$

- minimum required autonomy in performing daily activities with domestic tools, evaluated with the IADL [40].

Thirty-five voluntary participants living in the Peccioli area were involved in this experimentation; their age ranged from 65 to $85(73.8 \pm 6.0)$ years. Thirteen of them were male and 22 were female, and all of them were living independently in their own homes, some together with their partners and some alone. Everybody signed an informed consent.

\section{Procedure}

The experimentation phase was divided into three stages:

(1) the recruitment, based on the inclusion criteria, and preliminary questionnaire;

(2) the testing phase, in which the service was tested and evaluated by one elderly user at a time. First of all, the user was introduced to the three robots, and an instructive video about the potentiality of the RobotEra system was shown in order to arouse impressions about it. Then, the elderly participant was asked to perform the tasks of each service after it was introduced. During this phase, the user performed the tasks alone, and the observer helped him/her only if necessary. Finally, the user completed the questionnaire about Robot-Era robots and service acceptability;

(3) the final phase in which the acceptability of the whole Robot-Era system was evaluated.

Since user acceptability is influenced by the dependability of the system, technical data were also collected during the experimentation. The average duration of the shopping scenario was $18.04 \mathrm{~min}( \pm 4.32)$, while for the garbage scenario it was $8.1 \mathrm{~min}( \pm 2.36)$.

\section{Results}

Acceptability is defined as "the demonstrable willingness within a user group to employ technology for the tasks it is designed to support" [41], so an appropriate methodology has to be developed and applied in order to evaluate user acceptability.

Many studies have focused on the evaluation of assistive and companion robot acceptability within the elderly 
population, but only a few of them have focused on service robots. Several models have been developed to evaluate technology acceptance, but they are not always applicable for robots and elderly users. Since service robot acceptance by elderly people is an important factor in order to integrate service robotics into their daily lives, we applied an end-useroriented perspective, extracting some fundamental attributes from the UTAUT Model [42]. These attributes (usability, attitude, anxiety, trust and quality of life) are used to evaluate the Robot-Era robots and services. For our study, we constructed a questionnaire composed of open questions and statements, the response to which was based on a 5-point Likert scale [43] (1: Strongly Disagree, 2: Disagree, 3: No Opinion, 4: Agree and 5: Strongly Agree). Statements on general data such as age, gender, education and general attitude towards technology were also presented in a preliminary questionnaire.

The feedback provided by the elderly, collected by means of specific questionnaires and interviews, was used to investigate the degree of acceptability of Robot-Era services, and to measure some aspects of the dependability of the Robot-Era system. Statistics analysis was not applied, but data are shown with a qualitative approach.

\section{Acceptability of the Robot-Era Robots}

Regarding the acceptability of Robot-Era robots, elderly people's first impressions were collected using an ad hoc questionnaire based on a 5-point Likert scale. Data showed that the appearance of Doro was aesthetically pleasing for $77 \%$ of the participants and, in particular, $37 \%$ gave the maximum score. The appearance of Doro inspired confidence in $82 \%$ of the sample (51\%: 5 points and $31 \%: 4$ points). By $45 \%$ of elderly people, Doro was considered too big and bulky within a domestic environment.

Eighty-five per cent (51\%: 5 points and $34 \%: 4$ points) of the participants thought that Coro was aesthetically pleasing and its appearance inspired confidence in $88 \%$ of them (57\%: 5 points and $31 \%: 4$ points). Only $12 \%$ of them asserted that Coro was too big for a condominium environment. Finally, the aesthetics of Oro was appreciated by $71 \%$ of the elderly (34\%: 5 points and $37 \%: 4$ points), while a feeling of confidence was aroused in $78 \%$ of the participants (49\%: 5 points and $29 \%$ : 4 points).

Only $15 \%$ of elderly people thought that the dimensions of Oro were inappropriate for an urban environment. Table 1 summarises these results.

Acceptability of the Drug and Shopping Delivery Service

To investigate the usability of the service, the statement "I think that I would like to use this shopping delivery service frequently" was proposed and $26 \%$ of the participants replied with 3 points, $20 \%$ with 4 points and $29 \%$ with 5 points. Data showed that there was not a clear propensity to use this service because elderly people asserted that doing the shopping was a fun task and an opportunity to socialise. However, $71 \%$ of the participants believed that the drug and shopping delivery service was easy to use and, in particular, $54 \%$ of them agreed totally with the statement "I think this shopping delivery service was easy to use".

Evaluating the attitude towards this service as intention to use it, we determined that $86 \%$ of the participants would use the robots for doing shopping in case of need. Regarding the acceptability of the robots, $94 \%$ asserted that they were not nervous during performing the task and $63 \%$ of them agreed totally with the statement "I would trust in the robots' ability to perform the shopping service".

Finally, $66 \%$ of the participants (43\%: 5 points and $23 \%$ : 4 points) thought their independence would be improved by the use of the robots for shopping.

\section{Acceptability of the Garbage Collection Service}

Evaluating the usability of the service, $76 \%$ of the participants agreed totally with the statement "I think that I would like to use this garbage collection service frequently" and $82 \%$ with "I think the garbage collection service was easy to use". Data showed a very clear propensity to use this service because elderly people asserted that emptying the garbage is usually a boring task and with a robot for this purpose, they would have more free time for other activities. This was confirmed also by investigating the attitude towards the service: $86 \%$ would use the robots for garbage collection in case of need.

Data show that for $97 \%$ of the participants the service was easy to use and $80 \%$ of them agreed totally with the statement "I would trust in the robots' ability to perform the garbage service".

Finally, $77 \%$ of subjects (60\%: 5 points and $17 \%$ : 4 points) asserted that their quality of life would improve through the use of this garbage collection service. The results are summarised in Table 2.

From the point of view of the reliability of the system, the analysis focussed on the criticalities of the services, especially the ability to enter and exit the elevator and the ability to carry goods through the mechanism of exchange between Coro and Oro. Coro used the elevator twice for each scenario, and the success rate was $66.5 \%$ to enter the lift and $75 \%$ to exit it. The "exchange goods" task occurred once for each scenario, and the success rate for 35 experiments was $63 \%$.

\section{Conclusion}

In this paper, we described the development of the RobotEra system for providing shopping and garbage services at 
Table 1 Acceptability of the aesthetics of Robot-Era robots

\begin{tabular}{|c|c|c|c|c|c|c|c|c|c|c|c|c|c|c|c|}
\hline & Doro & & & & & Coro & & & & & Oro & & & & \\
\hline Likert scale & 1 & 2 & 3 & 4 & 5 & 1 & 2 & 3 & 4 & 5 & 1 & 2 & 3 & 4 & 5 \\
\hline Statement 1 & $3 \%$ & $3 \%$ & $17 \%$ & $40 \%$ & $37 \%$ & $0 \%$ & $0 \%$ & $14 \%$ & $34 \%$ & $51 \%$ & $3 \%$ & $9 \%$ & $17 \%$ & $37 \%$ & $34 \%$ \\
\hline Statement 2 & $3 \%$ & $0 \%$ & $14 \%$ & $31 \%$ & $51 \%$ & $3 \%$ & $0 \%$ & $9 \%$ & $31 \%$ & $57 \%$ & $3 \%$ & $3 \%$ & $17 \%$ & $29 \%$ & $49 \%$ \\
\hline \multirow[t]{2}{*}{ Statement 3} & \multicolumn{5}{|c|}{... a domestic environment } & \multicolumn{5}{|c|}{... a condominium environment } & \multicolumn{5}{|c|}{$\ldots$ an outdoor environment } \\
\hline & $24 \%$ & $6 \%$ & $26 \%$ & $24 \%$ & $21 \%$ & $56 \%$ & $15 \%$ & $18 \%$ & $6 \%$ & $6 \%$ & $80 \%$ & $3 \%$ & $3 \%$ & $6 \%$ & $9 \%$ \\
\hline
\end{tabular}

Statement 1: The appearance of the robot is aesthetically pleasing

Statement 2: The appearance of the robot inspires confidence in me

Statement 3: The robot is too big and bulky compared to...

Table 2 Attributes used for the assessment of the acceptability of Robot-Era services

\begin{tabular}{|c|c|c|c|c|c|c|c|c|c|c|c|}
\hline & \multirow[b]{2}{*}{ Likert scale } & \multicolumn{5}{|c|}{ Drug and shopping delivery } & \multicolumn{5}{|c|}{ Garbage collection } \\
\hline & & 1 & 2 & 3 & 4 & 5 & 1 & 2 & 3 & 4 & 5 \\
\hline \multirow[t]{2}{*}{ Usability } & Statement 1 & $11 \%$ & $14 \%$ & $26 \%$ & $20 \%$ & $29 \%$ & $0 \%$ & $0 \%$ & $15 \%$ & $9 \%$ & $76 \%$ \\
\hline & Statement 2 & $9 \%$ & $6 \%$ & $14 \%$ & $17 \%$ & $54 \%$ & $6 \%$ & $3 \%$ & $3 \%$ & $6 \%$ & $82 \%$ \\
\hline Attitude & Statement 3 & $3 \%$ & $3 \%$ & $6 \%$ & $3 \%$ & $86 \%$ & $3 \%$ & $3 \%$ & $3 \%$ & $6 \%$ & $86 \%$ \\
\hline Anxiety & Statement 4 & $94 \%$ & $3 \%$ & $0 \%$ & $0 \%$ & $3 \%$ & $97 \%$ & $0 \%$ & $0 \%$ & $0 \%$ & $3 \%$ \\
\hline Trust & Statement 5 & $0 \%$ & $0 \%$ & $9 \%$ & $29 \%$ & $63 \%$ & $0 \%$ & $0 \%$ & $6 \%$ & $14 \%$ & $80 \%$ \\
\hline Quality of life & Statement 6 & $20 \%$ & $0 \%$ & $14 \%$ & $23 \%$ & $43 \%$ & $11 \%$ & $0 \%$ & $11 \%$ & $17 \%$ & $60 \%$ \\
\hline
\end{tabular}

Statement 1: I think that I would like to use this service frequently

Statement 2: I think this service was easy to use

Statement 3: I would use the robots for performing this task in case of need

Statement 4: I was nervous performing this task with the robots

Statement 5: I would trust in the robots' ability to perform this task

Statement 6: I think my independence would be improved by the use of the robots for this task

home. Preliminary results of the experiments conducted with 35 elderly people are also shown. Both robots and services are analysed with an end-user-oriented perspective, considering the main attributes of acceptability.

The three robot platforms earned positive feedback from an aesthetical point of view, especially the condominium robot, inspiring confidence in the end-users. The domestic platform was considered too big for a normal apartment, and future improvements have to take this aspect into account.

In general, the proposed implementation was considered easy to use by most of the participants, demonstrating that the system is effective and adequate for the end-users' expectations. However, some difficulties occurred in terms of human-robot interaction, using the graphical user interface on the tablet and the natural language module that led to a low score in terms of usability. Indeed, due to the fact that some of the involved users used for the first time a tablet, they experienced some problems in using the touch screen graphical user interface. Additionally, elderly also experienced that the speech recognition module sometimes was not able to correctly recognise the vocal commands, and therefore, they were requested to repeat commands more times.

Reflection on the scenarios reveals an interesting outcome. In general, the garbage collection service was preferred over the shopping one. Usually, having to deal with the rubbish is not a pleasant task, while going out shopping represents an opportunity to socialise and have fun. In conclusion, while people would often use the garbage functionality, they would use the shopping service only if needed, as in case of illness.

Future work will focus on the general improvement of the whole system in terms of usability, reliability and robustness. In order to be able to better cope with a real domestic environment, manipulation features will be added to Doro. The system will also be extended to a large number of apartments, and scalability and privacy matters will be tackled.

Finally, according to all the aspects discussed in this paper and basing on the preliminary feedback given by end-users, the Robot-Era system has all the potentialities to 
be developed as a socially acceptable and believable provider of robotic services to elderly people.

Acknowledgments The research leading to these results has received funding from the European Community's Seventh Framework Programme (FP7/2007-2013) under Grant Agreement No. 288899 (Robot-Era Project)

\section{References}

1. McKinsey Global Institute. Disruptive technologies: advances that will transform life, business, and the global economy. McKinsey and Company, 2013.

2. Dario Paolo, Verschure PFMJ, Prescott Tony. Robot companions for citizens. Procedia Comput Sci. 2011;7:4751.

3. International federation of robotics. World Robotics 2013 Industrial Robots, 2013.

4. Eurostat. European social statistics-edition 2013, 2013.

5. Pereira A et al. iCat, the chess player: The influence of embodiment in the enjoyment of a game. Proceedings of the 7th international joint conference on autonomous agents and multiagent systems, vol 3. International Foundation for Autonomous Agents and Multiagent Systems, 2008.

6. Kidd CD, Taggart W, Turkle S. A sociable robot to encourage social interaction among the elderly. Proceedings of the 2006 IEEE international conference on robotics and automation (ICRA 2006). IEEE, 2006.

7. Stiehl WD, et al. The huggable: A therapeutic robotic companion for relational, affective touch. ACM SIGGRAPH 2006 emerging technologies. ACM, 2006.

8. Giraff Technologies company website: www.giraff.org.

9. Cesta A, Cortellessa G, Orlandini A, Tiberio L. Evaluating telepresence robots in the field. Agents Artif Intell Commun Comput InfSci. 2013;358:433-48.

10. iRobot/AVA company website: www.irobot.com/ava.

11. Ackerman E, Guizzo E. Mystery robot revealed: RoboDynamics Luna is fully programmable adult-size personal robot. IEEE Spectrum, 11 May 2011.

12. DOMEO project website: www.aal-domeo.eu.

13. Simonov Mikhail, Frisiello Antonella, Bazzani Marco. Using humanoid robot in ambient-assisted living. Global Telemed Health Updates Knowl Resour. 2012;5:438-42.

14. Lowet D, Frank H, Florence: A multipurpose robotic platform to support elderly at home. Proceedings of the 2012 workshop on ambient intelligence infrastructures (WAmIi), Nov 2012.

15. Ven A, Sponselee A, Schouten B. Robo M.D.: a home care robot for monitoring and detection of critical situations. European conference on cognitive ergonomics (ECCE 2010), Delft, The Netherlands, p. 375-376.

16. Badii A, et al. Companionable: graceful integration of mobile robot companion with a smart home environment. Gerontechnology. 2009;8(3):181.

17. Cavallo F, Aquilano M, Bonaccorsi M, Limosani R, Manzi A, Carrozza MC, Dario P. On the design, development and experimentation of the ASTRO assistive robot integrated in smart environments. 2013 IEEE international conference on robotics and automation (ICRA 2013), Karlsruhe, Germany, 6-10 May 2013.

18. Ferri G, et al. DustCart, an autonomous robot for door-to-door garbage collection: From DustBot project to the experimentation in the small town of Peccioli. 2011 IEEE international conference on robotics and automation (ICRA 2011), p. 655-660.
19. Kanda T, et al. An affective guide robot in a shopping mall. Proceedings of the 4th ACM/IEEE international conference on human robot interaction. ACM, 2009.

20. Chi G, et al. Robot collaboration through agent cooperation in a smart environment. 2012 IEEE/ASME international conference on advanced intelligent mechatronics (AIM). IEEE, 2012.

21. Veloso M, et al. Cobots: collaborative robots servicing multifloor buildings. 2012 IEEE/RSJ international conference on intelligent robots and systems (IROS). IEEE, 2012.

22. Robot-Era Project, http://www.robot-era.eu.

23. Bevilacqua R, Felici E, Marcellini F, Klemcke S, Nedopil C, Glende S, Filippo C, Aquilano M, Carrozza MC, Dario P. RobotEra project (FP7-ICT-2011.5.4): From the end-users perspective to robotics. preliminary findings. Proceedings of the AALambient assisted living forum 2012, Eindhoven, The Netherlands, 24-27 Sept 2012.

24. Quigley M, et al. ROS: An open-source robot operating system. ICRA workshop on open source software, 2009, vol. 3, No. 3.2.

25. Saffiotti A, et al. The PEIS-Ecology project: vision and results. 2008 IEEE/RSJ international conference on intelligent robots and systems (IROS 2008), p. 2329-2335.

26. Metralabs, http://www.metralabs.com.

27. Ferri, G, et al. DustCart, a mobile robot for urban environments: experiments of pollution monitoring and mapping during autonomous navigation in urban scenarios. 2010 IEEE international conference on robotics and automation (ICRA 2010) workshop on networked and mobile robot olfaction in natural, dynamic environments, Anchorage, AK, 7 May 2010.

28. Fox D, Burgard W, Thrun S. The dynamic window approach to collision avoidance. Robot Autom Mag. IEEE. 1997;4(1):23-33.

29. Dellaert F, Dieter F, Wolfram B, Sebastian T. Monte Carlo localization for mobile robots." Proceedings of the 1999 IEEE international conference on robotics and automation, vol. 2, 1999.

30. Samih E, Moreira A. Requirements and metrics for location and tracking for ambient assisted living. International conference on indoor positioning and indoor navigation (IPIN), 2012.

31. Gu Yanying, Lo Anthony, Niemegeers Ignas. A survey of indoor positioning systems for wireless personal networks. Commun Surv Tutor IEEE. 2009;11(1):13-32.

32. Blumenthal J, Grossmann R, Golatowski F, Timmermann D. Localization in ZigBee based sensor networks. 1st European ZigBee developers conference (EuZDC), Munchen-Dornach, Deutschland, 2007.

33. Wang Y, et al. Bluetooth positioning using RSSI and triangulation methods. 2013 IEEE consumer communications and networking conference (CCNC), 2013.

34. Whitehouse K, Karlof C, Culler D. A practical evaluation of radio signal strength for ranging-based localization. Mob Comput Commun Rev. 2007;11(1):41-52.

35. Di Rocco M, Pecora F, Saffiotti A. When robots are late: configuration planning for multiple robots with dynamic goals. IROS, 2013.

36. Dechter R, Meiri I, Pearl J. Temporal constraint networks. Artif Intell. 1991;49:61-95.

37. Di Rocco M, Pecora F, Saffiotti A. Closed loop configuration planning with time and resources. Proceedings of the ICAPS 2013 workshop on planning and robotics, 2013.

38. Cesta A, Oddi A, Smith SF. A constraint-based method for project scheduling with time windows. J Heuristics. 2002;8(1): 109-36.

39. Pfeiffer E. A short portable mental status questionnaire for the assessment of organic brain deficit in elderly patients. J Am Geriatr Soc. 1975;23(10):433-41.

40. Barberger-Gateau $\mathrm{P}$, et al. Instrumental activities of daily living as a screening tool for cognitive impairment and dementia in 
elderly community dwellers. J Am Geriatr Soc. 1992;40(11): 1129-34.

41. Dillon A. User acceptance of information technology. In: Karwowski W, editor. Encyclopedia of human factors and ergonomics. London: Taylor and Francis; 2001.

42. Venkatesh V, Morris MG, Davis GB, Davis FD. User acceptance of information technology: toward a unified view, MIS Quarterly, vol. 27, no. 3, pp. 425-478, 2003. http://www.jstor.org/stable/ 30036540 .

43. Likert R. A technique for the measurement of attitudes, Archives of psychology 140. New York, NY: Science Press; 1932. 